\title{
PROSTITUCIÓN Y CONFINAMIENTO: EL PUTERO 2.0
}

Águeda Gómez Suárez

Rosa $M^{a}$ Verdugo Matés

\section{Resumen}

En este artículo analizamos el comportamiento de los clientes de prostitución o "puteros" durante los meses de confinamiento derivados de la pandemia global que estamos padeciendo. Pese al riesgo para la vida y la salud, en estos meses la demanda de sexo comercial en España no ha cesado, tal y como se desprende de los testimonios de las mujeres en prostitución y de los contenidos de los chats de puteros. Este hecho contradictorio demuestra la fortaleza actual del mandato de masculinidad patriarcal en España - primer país europeo en consumo de prostitución - y la hegemonía de un modelo de masculinidad fallido en su humanidad, incompatible con el momento de vulnerabilidad total que estamos sufriendo como sociedad.

Palabras claves: Masculinidad, cliente de prostitución, prostitución, confinamiento, pandemia.

\section{Resumo}

\section{Prostitucão e confinamento: o putanheiro 2.0}

Neste artigo analisamos o comportamento dos clientes de prostituição ou "puteros" durante os meses de confinamento da pandemia global que estamos a viver. Apesar do risco para a vida e a saúde, nestes meses a procura de sexo comercial em Espanha não cessou, como se pode ver pelos testemunhos de mulheres na prostituição e pelos conteúdos dos chats dos clientes de sexo pago. Este facto contraditório demonstra a força atual do mandato patriarcal da masculinidade em Espanha - o primeiro país europeu em consumo

Facultad de Ciencias de la Educación (Ourense), Universidad de Vigo, 32004 Ourense, España. Dirección postal: Facultad de Ciencias de la Educación, Universidad de Vigo - R/ Doctor Temes, s/n, 32004 Ourense, España.

Correo electrónico: agueda@uvigo.es

** Facultade de Ciencias Económicas e Empresariais, Universidade de Santiago de Compostela, 15782 Santiago de Compostela, España.

Dirección postal: Facultade de Ciencias Económicas e Empresariais, Universidad de Santiago de Compostela - Avda. do Burgo das Nacións, s/n. Campus Norte - 15782 Santiago de Compostela, España.

Correo electrónico: rosa.verdugo@usc.es 
de prostituição - e a hegemonia de um modelo de masculinidade que falhou na sua humanidade, incompatível com o momento de vulnerabilidade total que estamos a sofrer como sociedade.

Palavras-chave: Masculinidade, cliente de prostituição, prostituição, confinamento, pandemia.

Abstract

Prostitution and Confinement: The Whorer 2.0

In this article we analyze the behavior of prostitution clients, or "whorers", during the months of confinement derived from the global pandemic that we are experiencing. Despite the risk to life and health, the demand for commercial sex in Spain has not ceased in recent months, as can be seen from the testimonies of women in prostitution and the contents of the chat rooms of prostitution clients. This contradictory fact demonstrates the current strength of the patriarchal masculinity mandate in Spain - the first European country in the consumption of prostitution - and the hegemony of a failed model of masculinity in its humanity, incompatible with the moment of total vulnerability that we are suffering as a society.

Keywords: Masculinity, prostitution clients, prostitution, confinement, pandemic.

\section{Introducción}

La prostitución es una pieza importante del crimen organizado, solo por detrás de la droga en cuanto a su alcance y a la cantidad de dinero que genera. El sitio web Havocscope estima que los ingresos mundiales procedentes de la prostitución rondan los 186 billones de dólares estadounidenses al año, ocupando España el segundo puesto con 26,5 billones ${ }^{1}$. Lógicamente, este elevado volumen de negocio está asociado a un también elevado consumo de sexo comercial, tal y como evidencian diferentes estudios. Así, según una investigación realizada a finales de la década de 1990 en once países europeos, en España cuatro de cada diez varones mantuvieron alguna vez relaciones sexuales de pago, ocupando este país el primer puesto de Europa en el consumo de prostitución (Hubert et al. 1998). En investigaciones realizadas por organismos oficiales españoles también se constata un elevado consumo de sexo en contextos prostitucionales. En concreto, en una encuesta elaborada por el Instituto Nacional de Estadística (INE) y el Ministerio de Sanidad y Consumo se indica que, en el año 2003, el 27,3\% de los hombres españoles pagaron alguna vez por servicios de prostitución (INE 2004). Posteriormente, en un estudio realizado por el Centro de Investigaciones Sociológicas (CIS) se señala que, en 2008, un 32,1\% de los varones españoles mayores de 16 años recurrió alguna vez al sexo de pago (CIS 2009). Además, España no sólo es el país europeo con mayor consumo de servicios de prostitución, sino que también es el primer

Estos y otros datos relativos a la prostitución en diferentes países del mundo están disponibles en https:/ / havocscope.com/prostitution-statistics / 
destino tanto de las víctimas destinadas a la explotación sexual (APRAMP 2005; APRAMP 2011; Ranea 2019) como de las víctimas de trata (APRAMP 2016; APRAMP 2018), operando ciertas zonas del territorio español como destinos del turismo sexual.

La prostitución es un fenómeno "generizado" ya que la casi totalidad de los demandantes de sexo comercial son hombres y la inmensa mayoría de las personas en prostitución son mujeres. En este sentido, la prostitución es una realidad en donde se manifiesta una ideología contraria a los principios de igualdad entre hombres y mujeres (Pateman 1995; MacKinonn 1998), y que institucionaliza una de las formas más exacerbadas de violencia de género. En este sentido, Beatriz Gimeno define la prostitución como una institución que estructura el patriarcado con el objetivo principal de mantener u ordenar la desigualdad sexual (Gimeno 2018). Además, la prostitución es una institución histórica que cambia al mismo tiempo que lo hace la sociedad, registrándose importantes modificaciones en esta fase del capitalismo neoliberal (García Massagué 2009; Gimeno 2012; Cobo 2017). De hecho, el "capital sexual" se ha convertido en una mercancía donde los individuos, principalmente las mujeres, transforman en mercancía su apariencia y sus atributos para integrarse y sobrevivir. Asistimos la denominada "pornificación de la cultura" en el mundo (Illouz 2020; Illouz y Kaplan 2020), ya que la pornografía se convierte en una narrativa social generalizada.

En el actual contexto pandémico, el confinamiento domiciliario severo o parcial, la distancia de seguridad, el fin de las aglomeraciones en espacios de ocio, etc., están suponiendo un punto de inflexión en las relaciones humanas. Sin embargo, y pese al riesgo para la salud y para la vida de las personas implicadas, la demanda de prostitución en España no cesó, tal y como se desprende de los testimonios de las mujeres en prostitución y de los contenidos de los chats de puteros. De hecho, a raíz de la pandemia del COVID-19, una de las temáticas de la agenda mediática fue la situación de la industria sexual "confinada" y de los riesgos de los espacios prostitucionales para la salud pública.

En este artículo analizamos la masculinidad patriarcal, y más específicamente la masculinidad patriarcal confinada, ya que resulta una pieza clave en la reproducción del patriarcado y de la violencia contra las mujeres. En concreto, el centro de nuestro análisis se reduce a uno de los actores fundamentales del fenómeno de la prostitución: los clientes, puteros o prostituidores, figura que, a pesar de su importancia en el mundo de la prostitución, sólo es tratada en el 1\% de la producción científica al respecto (Ranea 2019). Pero antes de llevar a cabo este estudio revisamos la literatura académica sobre el cliente de prostitución en España, lo que nos permitirá visualizar las continuidades y las rupturas del comportamiento del putero antes y después de la pandemia. 


\section{Literatura científica sobre el putero español}

El primer trabajo que analizó al cliente de prostitución en España fue elaborado por $\mathrm{M}^{\mathrm{a}}$ José Barahona y Luis Mariano García. A partir de la información recopilada en 15 entrevistas a clientes de prostitución de las calles de Madrid, extrajeron cinco motivos alegados por estos hombres para recurrir al sexo de pago: la insatisfacción de las relaciones sexuales afectivas con sus parejas; la necesidad de obtener una mayor frecuencia y variedad de relaciones sexuales; el poder ejercitado por el hecho de pagar servicios sexuales; el deseo de satisfacer fantasías sexuales; y por último, la búsqueda de diversión, especialmente como experiencia grupal de varones (Barahona y García 2003).

Rafael López y David Baringo realizaron 12 entrevistas a diferentes perfiles de hombres consumidores de prostitución en Zaragoza. A partir de los datos obtenidos establecieron seis categorías de clientes: hombres a los que les costaba relacionarse con mujeres; varones que salían en grupo para divertirse; hombres casados que deseaban tener relaciones extramaritales como infidelidad menor; varones que tenían crisis de pareja y buscaban en el pago de servicios sexuales cierta venganza; hombres para los cuales el pago de servicios sexuales surgían como un elemento del ámbito laboral o de los negocios; y, por último, varones jóvenes que pagaban servicios sexuales porque les suponía una menor complicación y un menor esfuerzo (López y Baringo 2006).

La Asociación Askabide elaboró un estudio para definir el perfil del consumidor de prostitución en Vizcaya, recogiendo la información a través de formularios entregados a 94 clientes. Los resultados indicaron que dentro de los demandantes de sexo de pago predominaban los hombres solteros de entre 30 y 40 años, siendo las principales motivaciones para demandar este servicio la búsqueda de sexo sin compromiso y el deseo de realizar prácticas sexuales diferentes a las que realizaban con sus parejas (Asociación Askabide 2008).

El sociólogo Hilario Sáez y su equipo desarrollaron para la Fundación Iniciativa Social una investigación, aún inédita, titulada ¿Cómo somos los hombres? Masculinidades y consumo de prostitución en Andalucía. En este trabajo analizan los discursos de hombres andaluces de diferentes clases sociales y edades mediante el uso de la técnica del grupo de discusión, presentando un panorama socioambiental de las actitudes hacia el fenómeno de la prostitución (Sáez et al. 2015).

Uno de los pocos trabajos de carácter cuantitativo destacables es el realizado por Carmen Meneses. A partir de una muestra de 138 clientes que cubrieron cuestionarios autogestionados, identificó las principales motivaciones de estos hombres para acudir al sexo de pago y las principales prácticas sexuales solicitadas por ellos. De las catorce razones para consumir prostitución que aparecían en el cuestionario, las tres con las que los clientes estaban en mayor acuerdo fueron: poder elegir a distintas mujeres; pensar que el sexo de pago genera menos problemas; y tener sexo rápido e impersonal (Meneses 2010). 
Águeda Gómez y Silvia Pérez publicaron los resultados de una investigación en la que se analizan los relatos de hombres demandantes de sexo comercial en Galicia (Gómez y Pérez 2009)² y, posteriormente, ampliaron este estudio al resto de España (Gómez et al. 2015)3. En ambas investigaciones se concluye la inexistencia de un perfil sociológico concreto entre el grupo de hombres que pagaron por tener sexo con mujeres, ya que entre los clientes de prostitución aparecieron representadas todas las edades, clases sociales, etnias, niveles formativos, ocupaciones, ideologías políticas y situaciones sentimentales. Sin embargo, sí que se identificaron distintos acentos discursivos en la narrativa del putero. En concreto, se reconocieron cuatro relatos que en una escala gradual de subhumanización de las mujeres fueron: relato misógino (cosifica a la mujer), relato consumidor (compra lo que se vende), relato amigo (empatiza pero no politiza) y relato crítico (arrepentido) (Gómez et al. 2015).

A continuación, en el siguiente sección mostramos algunas continuidades y algunas rupturas que se observan en el comportamiento del putero poscovid.

\section{El putero poscovid: continuidades y rupturas}

Los perfiles discursivos de los clientes de sexo de pago muestran su continuidad en el putero poscovid, tal y como se desprende de los resultados aproximativos que se obtienen a partir del análisis de los relatos extraídos de los chats de puteros, de las entrevistas informales con clientes de prostitución y de los testimonios de mujeres en prostitución y de las ONGs que las atienden.

Ahora bien, la puesta en marcha del estado de alarma, el confinamiento y la reducción de los aforos en los diferentes negocios modificaron nuestras relaciones sociales. Así, patrones de sociabilidad masculina como las cenas de empresa, las despedidas de soltero o la última copa - que en bastantes ocasiones finalizaban en los clubs nocturnos - han experimentado una reducción considerable, lo que ha disminuido el consumo grupal de sexo comercial en estos locales. Sin embargo, se detecta un incremento del consumo individualizado de sexo de pago en pisos particulares controlados por la industria sexual. También se observa un auge del consumo de sexo virtual, destacando el incremento de las videollamadas con webcams

En esta investigación se realizaron 17 entrevistas a clientes, 5 entrevistas a grupos de discusión de colectivos masculinizados (médicos, sindicalistas del metal y estudiantes de ciclo formativo de electricidad, informática y secundaria), 6 entrevistas a mujeres en prostitución, 2 entrevistas a transexuales en prostitución, 3 a dueños y gerentes de clubes de alterne, 3 a técnicos de servicios sociales que trabajan con mujeres en prostitución y a 1 trabajador de un club.

En esta investigación se aplicaron entrevistas en profundidad semidirigidas a 29 clientes, 13 mujeres en prostitución, 3 dueños de clubes y/o pisos, 4 trabajadores de clubes y/o pisos y 23 técnicos sociales, académicas y/o profesionales de los servicios sociales. Además, se llevaron a cabo tres entrevistas grupales a grupos de hombres en nueve comunidades autónomas españolas. 
y de la porno-prostitución. En estas nuevas prácticas en streaming el putero on line paga para que otro individuo materialice sus deseos sexuales con la mujer prostituida. Las nuevas tecnologías permiten que este negocio llegue cada vez más lejos. En efecto, se ha extendido la figura de la webcamer, mujer que hace lo que el cliente les pide, a veces acompañada por otra mujer, a veces por un hombre (o más), que actúan a modo de alter ego del cliente on line. Y el putero puede tener un show privado si paga más (Lozano y Conellie 2020). Como señala la investigadora y activista Mabel Lozano,

Un hombre puede entrar en una sala de una mujer de Filipinas y pedir que la violen, que se meta de todo... Los hombres entran en línea y les piden que se metan martillos por el ano y la vagina, que se pongan pinzas en el pecho... Y eso no es virtual, es real. (ibidem)

Si bien es cierto que la pornografía siempre actuó a modo de marketing del negocio prostitucional, en la actualidad la frontera entre el mundo de la prostitución y el mundo de la pornografía se está desvaneciendo. El consumo de porno-prostitución ha aumentado, y para satisfacer esta creciente demanda se captan a mujeres y niñas para hacer porno amateur en internet a través de aplicaciones tan extendidas como Onlyfans, IsMyGirl, Manyvids, Justforfans, Tinder, IWantFanClub y otras similares. Hoy en día muchas mujeres adolescentes y jóvenes hacen uso de estas opciones para ganar algo de dinero y después se ven en una espiral de la que resulta difícil salir: la "ciberdelincuencia" chantajea y "sextorsiona" a estas mujeres con la difusión de sus vídeos ante entornos comprometidos si se niegan a seguir generando contenidos similares. Este es el aparato publicitario de la industria sexual: la pornografía mainstream que empresas como Pornhub ofertaron gratuitamente al inicio del confinamiento.

Es indudable que el impacto del confinamiento se ha traducido en un incremento de la vulnerabilidad y explotación de las mujeres en prostitución. Estas mujeres han sufrido situaciones de extrema necesidad alimentaria y habitacional $\mathrm{y}$, al mismo tiempo, han visto como se incrementaban sus deudas con tratantes, proxenetas y explotadores, lo que las ha obligado a aceptar prácticas con clientes que en tiempos precovid nunca habrían aceptado. El contraste que se produce entre la banalización de la demanda de sexo comercial y el dramatismo de la situación de las mujeres en prostitución invita a la reflexión en torno al fenómeno de la prostitución, y en dicha reflexión la perspectiva de género, la feminización de la pobreza y la denominada "pedagogía de la crueldad" (Segato 2016) deberían ocupar un papel central.

En España, los estudios que se centran en el análisis de los clientes de prostitución comparten conclusiones muy similares, coincidiendo en señalar el papel hegemónico de la masculinidad patriarcal que pivota en una idea hipersexual de la virilidad (Connell 2003; De Miguel 2015) y que está en contra de la equidad de 
género (Granxeiro 2017). Una de las conclusiones de estas investigaciones es que los consumidores de prostitución no buscan placer sexual, sino una motivación político-identitaria para obtener el status de "hombría" o, por lo menos, para parecer "hombres" ante el grupo de pares. El hecho social de ir a un espacio de prostitución se entiende como un acto expresivo dirigido, por un lado, al grupo de pares masculinos que examinan su grado de virilidad y, por otro lado, a las mujeres para recordarles que en el orden patriarcal ellas son las dominadas, explotadas y cosificadas. La subyugación del llamado "tributo femenino" permite al hombre ser reconocido y titulado como "sujeto masculino" (Segato 2014).

Pero, ¿qué ocurre cuando la sensación de vulnerabilidad vital de todas y todos se instala en nuestras vidas debido a una pandemia de dimensiones globales? ¿Qué ocurre con los puteros? En esta situación, la persistencia de estos modelos de masculinidad depredadora se convierte en un síntoma de anomalía social: hombres que siguen consumiendo sexo comercial a pesar de los riesgos para su salud y para su vida (y la de otras personas). La masculinidad patriarcal confinada ha resultado ser temeraria, irresponsable, egoísta e insolidaria, y continúa pivotando en torno a una hipersexualidad denominada por el patrón del "vividor-follador". Este hecho demuestra la fortaleza actual del mandato de masculinidad tóxica y la hegemonía de un modelo de hombría fallida en su humanidad, incompatible con el momento de vulnerabilidad vital que estamos sufriendo como sociedad.

\section{Hombres en busca de identidad}

El sociólogo Pierre Bourdieu afirmó que el patriarcado fabrica hombres machistas mediante un sistema de dominación masculina que actúa como ideología hegemónica, que comporta una dimensión simbólica de jerarquías donde el dominador (el hombre) debe conseguir obtener de la dominada (la mujer) una forma de adhesión inconsciente, inmediata y pre-reflexiva de los cuerpos socializados (Bourdieu 2000). En este sentido, las categorías "masculino" y "femenino" se erigen como categorías políticas (Badinter 1993; Castells y Subirats 2007; Cobo 2020).

El contexto neoliberal y la "pedagogía de la crueldad" inoculan la falta de empatía en las relaciones humanas a partir de patrones educativos de carácter sexoafectivo entre los principales agentes de la socialización, caracterizados por una semiótica sexista y una pornografía misógina que atraviesa la cultura mainstream globalizada (Gabriel 2017; Alario 2018; Ballester y Orte 2019). Así, según Beatriz Gimeno diversos estudios muestran que las primeras experiencias sexuales de hombres con prostitutas, lejos de ser satisfactorias, son traumáticas, y en ellas el hombre aprende a disciplinar el miedo, el asco, la falta de empatía y a tener una erección sin importar la apetencia, siendo este el camino de la exaltación de la masculinidad (Gimeno 2018). 
En la actualidad, gran parte del colectivo masculino ha perdido el poder económico, el poder en el seno de la familia y también el poder sexual. La vieja masculinidad hegemónica construida en base a la idea del padre de familia proveedor y protector está siendo sustituida por el "macho herido", que se expresa en una hombría agresiva, machista y violenta, y que desdeña la autonomía de las mujeres como si se tratara de un ataque hacia los hombres. En consecuencia, el consumo de servicios de prostitución pervive al abrigo de un emergente modelo de identidad masculina patriarcal, el conocido como "vividor-follador", que trata de resistirse a la pérdida de otro de los espacios hegemónicos del pasado: el poder sexual. La saña expresada en actos de violencia extrema muestra una hipermasculinidad (hombres-monstruos) que controla, domina y a veces destruye el territorio-cuerpo de la mujer a modo de víctima propiciatoria (Girard 2005). En este sentido, la misoginia del putero hacia las prostitutas, y del que hay constancia en los foros y redes sociales, es un odio contra todas las mujeres que los hombres no pueden expresar en su vida cotidiana (Gimeno 2018). Es así como el patriarcado fabrica hombres machistas, misóginos y puteros.

El acceso al porno, bien de forma intencionada o casual, se hace cada vez a una edad más temprana. La pornografía es más accesible y más agresiva que nunca, por lo que los niños de hoy están siendo moldeados en sus subjetividades y en sus gustos eróticos por esta escuela de sexualidad en la que se ha convertido el porno maistream, cuyo mensaje pedagógico principal es el de la crueldad patriarcal y misógina: el hombre es el que manda y el comportamiento de la mujer se adapta a lo que él pide. La pornografía va de poder, no de placer (Cobo 2020). La producción de la masculinidad en el contexto patriarcal y su relación con el consumo de sexo comercial se construye en referencia a los otros hombres y a un mandato de masculinidad cruel, que encuentra en el sexo comercial una oportunidad de realización identitaria. En tiempos de COVID-19, todas estas muestras de virilidad patriarcal (legal, cultural, simbólica y subjetiva) confirman el fallido proyecto de humanización civilizatoria de la masculinidad patriarcal, embrutecida y misógina. El consumo elevado de pornografía mainstream, brazo ideológico de esta estrategia de crueldad, resulta un ejemplo de la actual fortaleza del mandato de masculinidad patriarcal destinado a los varones, pese a los empujes sociales civilizatorios de la democracia, los derechos humanos y el feminismo. En este contexto de violencia contra las mujeres, la pornografía mainstream oferta mujeres cada vez más vulnerables para ser humilladas y torturadas.

\section{Conclusiones}

Durante la pandemia que asola al mundo, se ha podido observar como en España - primer país europeo consumidor de sexo comercial - la industria sexual se ha replegado a nuevos espacios más discretos y de mayor clandestinidad para 
seguir ofertando sexo de pago. Si bien es cierto que el volumen de actividad sufrió una considerable reducción, también es innegable que la demanda de este tipo de servicios se ha canalizado a través de dos vías principales: por un lado, el consumo intensivo de pornografía mainstream y de porno-prostitución y, por otro lado, la organización de fiestas privadas y la ampliación de la oferta desde pisos controlados por la industria sexual.

Pero, ¿cómo es posible que pese al momento de vulnerabilidad vital y sanitaria, continuara la demanda de sexo comercial por parte de un número considerable de hombres? ¿Qué tipo de modelos socioeróticos y de masculinidad sustentan este tipo de actitudes y conductas? Para entender esta contradicción se debe acudir a explicaciones que implican partir del fuerte mandato de masculinidad hegemónica que determina la conducta de estos hombres. La fragilidad de la identidad masculina depende de este tipo de acciones depredadoras y crueles para con otros seres humanos (Girard 2005), en este caso las mujeres en prostitución. Ante la percepción de una virilidad amenazada, se opta por arriesgar la propia salud, la de sus entornos familiares y la de las propias mujeres en prostitución. El mandato expresivo de lo viril se impone frente a la racionalidad de la supervivencia humana y del bien común (Segato 2016).

La poderosa industria patriarcal de la cultura y el ocio (De Miguel 2015; Sáez et al. 2015) derivó la centralidad del espectáculo en la cosificación del cuerpo de las mujeres como una diversión más, pero a lo que realmente se asiste es a una tortura. En el actual contexto de neoliberalismo sexual, toda relación humana es susceptible de convertirse en una mercancía: la vida se transforma en cosa (de Miguel 2015, de Miguel et al. 2017; Cobo 2018; Segato 2019). En tiempos de COVID-19, las lógicas de la crueldad neoliberal pugnan frente a las lógicas del cuidado derivadas de la situación de fragilidad vital que todas y todos hemos experimentado. En estas circunstancias, la industria sexual se recicla apostando por la porno-prostitución, nuevas prácticas de explotación sexual en streaming en las que el putero on line paga para ver como otro individuo realiza con la mujer en prostitución las prácticas sexuales que él ordena.

La "pedagogía de la crueldad" y sus brazos ideológicos en esta estrategia de la monstruosidad, como la pornografía mainstream, programa a las personas para la baja sensibilidad, para la baja empatía hacia el sufrimiento ajeno, hacia un individualismo y un encapsulamiento muy cercano a lo que Hanah Arendt identifica con el totalitarismo (Arendt 2006), y que permite funcionar adecuadamente en ese orden consumidor y cosificador de vida (Bauman 2015). En efecto, el sistema prostitucional funciona como fórmula totalitaria de disciplinamiento a través de las redes criminales de trata de mujeres y niñas con fines de explotación sexual, y también mediante la organización de los espacios prostitucionales como campos de concentración donde se castiga y disciplina a las mujeres a través de amenazas, multas, sanciones, extorsiones y controles que acaban erosionando su dignidad (Galindo y Sánchez 2007; Atencio 2015; Granxeiro 2017; Tiganus 2017). 
Las interacciones sexuales de nuestro tiempo están marcadas por ese sentimiento incierto. A diferencia de lo que sucedía hasta no hace tanto, hoy ya no se sabe cuáles son las normas que regulan esas relaciones, ni cuál es su objetivo preciso. Esta pandemia está generando una nueva forma de socialización más individualista, menos humanizada y menos empática. El reto actual al que se enfrenta la humanidad consiste en instalar otra "gramática de la socialidad" que desmonte el mandato de masculinidad hegemónica cruel y letal para poder iniciar un nuevo contrato sexual a través del camino de los afectos que trabaje a favor de la equidad, la justicia y de los derechos humanos.

\section{Referencias bibliográficas}

Alario Gavilán, Mónica. 2018. “La influencia del imaginario de la pornografía hegemónica en la construcción del deseo sexual masculino prostituyente: un análisis de la demanda de prostitución." Asparkía: Investigació feminista 33: 61-79. DOI: http:/ / dx.doi.org/10. 6035 / Asparkia.2018.33.4

APRAMP (Asociación para la Prevención y Reinserción de la Mujer Prostituida). 2005. La prostitución, claves básicas para reflexionar sobre el problema. APRAMP/Fundación Mujeres. Disponible en http://www.mujeresenred.net/IMG/pdf/prostitucion-claves_ basicas.pdf (consultado 04/05/2020).

APRAMP (Asociación para la Prevención y Reinserción de la Mujer Prostituida). 2011. La trata con fines de explotación sexual. [s.1.]: APRAMP. Disponible en http: / / www.apramp. org / upload/doc166_LATRATA.pdf (consultado 04/05/2020).

APRAMP (Asociación para la Prevención y Reinserción de la Mujer Prostituida). 2016. A pie de calle. Actuaciones con menores víctimas de trata. APRAMP/Fundación Mujeres. Disponible en https: / / apramp.org/ download/a-pie-de-calle-actuaciones-con-menoresvictimas-de-trata/ (consultado 04/05/2020).

APRAMP (Asociación para la Prevención y Reinserción de la Mujer Prostituida). 2018. Memoria de actividades. APRAMP/Fundación Mujeres. Disponible en https:/ / apramp. org / wp-content/ uploads / APRAMP.Memoria.2018.pdf (consultado 04/05/2020).

Arendt, Hannah. 2006. Los orígenes del totalitarismo. Madrid: Alianza.

Asociación Askabide. 2008. Perfil de clientes de prostitución en Bizkaia. Bilbao: Mensajero.

Atencio, Graciela. 2015. Feminicidio. Madrid: La Catarata.

Badinter, Élisabeth. 1993. XY: La identidad masculina. Madrid: Alianza.

Ballester Brage, Lluís y Carmen Orte Socías. 2019. Nueva pornografía y cambios en las relaciones personales. Barcelona: Editorial Octaedro.

Barahona Gomariz, Ma José, y Luis Mariano García Vicente. 2003. Una aproximación al perfil del cliente de prostitución femenina en la Comunidad de Madrid. Madrid: Dirección General de la Mujer.

Bauman, Zygmunt. 2015. Ceguera moral. La pérdida de sensibilidad en la moral líquida. Barcelona: Paidós.

Bourdieu, Pierre. 2000. La Dominación Masculina. Barcelona: Anagrama.

Castells, Manuel, y Marina Subirats. 2007. Mujeres y hombres: un amor imposible. Madrid: Alianza.

Centro de Investigaciones Sociológicas (CIS). 2009. Distribuciones marginales. Encuesta nacional de salud sexual. Estudio n ${ }^{\circ}$ 2.780. Noviembre 2008 - Enero 2009. Disponible en http: / / www.cis.es/ cis / opencms /-Archivos / Marginales /2780_2799/2780/ES2780.pdf 
Cobo Bedía, Rosa. 2017. La prostitución en el corazón del capitalismo. Madrid: La Catarata

Cobo Bedía, Rosa. 2018. "El imaginario pornográfico como pedagogía de la prostitución." Oñati Socio-legal Series 9 (S1), S6-S26. DOI: https:/ / doi.org/10.35295/osls.iisl/00000000-0000-1002

Cobo Bedía, Rosa. 2020. Pornografía: el placer del poder. Madrid: Ediciones B.

Connell, Robert. 2003. Masculinidades. Trad. Irene $\mathrm{M}^{\mathrm{a}}$ Artigas. México: Universidad Nacional Autónoma de México.

De Miguel, Ana. 2015. Neoliberalismo sexual. Madrid: Cátedra.

De Miguel, Ana, y Laura Nuño Gómez (eds.). 2017. Elementos para una teoría crítica del sistema prostitucional. Granada: Comares.

Gabriel, Karen. 2017. Power of Porn Cultures [online]. Disponible en https:/ / www.tni.org/ files / publication-downloads/ stateofpower2017-porn-cultures.pdf

Galindo, María, y Sonia Sánchez. 2007. Ninguna mujer nace para puta. Buenos Aires: Lavaca.

García Massagué, Mónica. 2009. Historia de los burdeles. Barcelona: Editorial Océano.

Gimeno, Beatriz. 2012. La prostitución. Barcelona: Bellaterra.

Gimeno, Beatriz. 2018. "La nueva utilidad de la prostitución en el neoliberalismo." Atlánticas: Revista Internacional de Estudios Feministas 3(1): 13-32. DOI: https://doi.org/ 10.17979/arief.2018.3.1.3077

Girard, René. 2005. La violencia y lo sagrado. Madrid: Editorial Anagrama.

Gómez Suárez, Águeda, y Silvia Pérez Freire. 2009. Prostitución: clientes e outros homes. Vigo: Edicións Xerais.

Gómez Suárez, Águeda, Silvia Pérez Freire, y Rosa Verdugo Matés. 2015. El putero español: quienes son y qué buscan los clientes de prostitución. Madrid: La Catarata.

Granxeiro, Carmen. 2017. Benvidas ao club. Disponible en https:/ / vimeo.com/290154154

Hubert, Michel, Nathalie Bajos, and Theo Sandfort. 1998. Sexual Behavior and HIV/AIDS in Europe: Comparisons of National Surveys. London: UCL Press.

Illouz, Eva. 2020. El fin del amor. Una sociología de las relaciones negativas. Madrid: Editorial Katz.

Illouz, Eva, y Dana Kaplan. 2020. El capital sexual en la modernidad tardía. Barcelona: Herder.

Instituto Nacional de Estatística (INE) y Ministerio de Sanidad y Consumo. 2004. Salud y Hábitos sexuales. Las conductas sexuales desde la perspectiva del sida. Madrid: Instituto Nacional de Estadística.

López, Rafael, y David Baringo. 2006. Nadie va de putas, el hombre y la prostitución femenina. Zaragoza: Organización Editorial S.L.

Lozano, Mabel, y Pablo Conellie. 2020. Pornoexplotación. Madrid: Editorial AlRevés.

MacKinnon, Catherine. 1998. Crímenes de guerra. Crímenes de paz. Los derechos humanos, las conferencias Oxford Amnesty de 1993. Madrid: Editorial Trotta.

Meneses, Carmen. 2010. "Factores motivacionales en una muestra de hombres españoles que pagan por servicios sexuales." Revista de la Asociación Española de Neuropsiquiatría 30 (107): 393-407.

Pateman, Carole. 1995. El contrato sexual. Barcelona: Anthropos.

Ranea Triviño, Beatriz. 2019. “Masculinidad (hegemónica) resquebrajada y reconstrucción subjetiva en los espacios de prostitución." Oñati Socio-legal Series 9 (extra 1): 61-81. DOI: https: / / doi.org/10.35295/osls.iisl/0000-0000-0000-1005

Sáez, Hilario et al. (2015). Cómo somos los hombres: Masculinidades y consumo de prostitución en Andalucía. Sevilla: Fundación Iniciativa Social. (Texto inédito).

Segato, Rita. 2014. "El sexo y la norma: frente estatal, patriarcado, desposesión, colonidad." Revista de Estudos Feministas 22(2): 593-616. DOI: https://doi.org/10.1590/S0104026X2014000200012 
Segato, Rita. 2016. La guerra contra las mujeres. Madrid: Traficantes de Sueños.

Segato, Rita. et al. 2019. "Entrevista a Rita Laura Segato." In Feminismos a la contra. Entre-vistas al Sur global, organizado por Luis Martínez Andrade, 71-88. Santander: La Vorágine, editorial crítica (Otramérica).

Tiganus, Amelia. 2017. "La revuelta de las putas." Feminicidio.net - 27/10/2017. Disponible en https: / / feminicidio.net/ articulo/la-revuelta-las-putas

Águeda Gómez Suárez. Doctora en Sociología por la Universidad Complutense de Madrid y profesora titular en la Facultad de Ciencias de la Educación de la Universidad de Vigo. Intereses de investigación: prostitución, sistemas sexo/género y etnicidad. Publicaciones recientes: Gómez Suárez, A. (2020). “La ‘pedagogía de la crueldad en el relato 〈putero〉." Oxímora: revista internacional de ética y política 16: 122-136.

Correo electrónico: agueda@uvigo.es

Rosa $\mathbf{M}^{\mathrm{a}}$ Verdugo Matés. Doctora en Ciencias Económicas y Empresariales por la Universidad de Santiago de Compostela y profesora en el Departamento de Economía Aplicada de la misma universidad. Intereses de investigación: prostitución, migraciones, desarrollo económico. Publicaciones recientes: Verdugo Matés, R.M. et al. (2019). "Determinantes de la participación femenina en el mercado de trabajo en la Galicia rural y urbana de 1924." Historia agraria: Revista de agricultura e historia rural 79: 161-186.

Correo electrónico: rosa.verdugo@usc.es

Artículo recibido el 04 de Enero y aceptado para su publicación el 22 de Marzo de 2021. 